This item was submitted to Loughborough's Research Repository by the author.

Items in Figshare are protected by copyright, with all rights reserved, unless otherwise indicated.

\title{
Development of lower limb rehabilitation evaluation system based on virtual
} reality technology

\section{PLEASE CITE THE PUBLISHED VERSION}

http://dx.doi.org/10.1109/RCAR.2016.7784083

PUBLISHER

(C) IEEE

VERSION

AM (Accepted Manuscript)

LICENCE

CC BY-NC-ND 4.0

\section{REPOSITORY RECORD}

Shen, Shihui, Chang Gao, Yong Zhao, Haojian Lu, Yajing Shen, Chunbao Wang, Tongyang Sun, et al.. 2019. "Development of Lower Limb Rehabilitation Evaluation System Based on Virtual Reality Technology". figshare. https://hdl.handle.net/2134/25946. 


\title{
Development of Lower Limb Rehabilitation Evaluation System Based on Virtual Reality Technology *
}

\author{
Shihui Shen, Chang Gao, Yong Zhao, Haojian Lu, Yajing Shen, Chunbao Wang, Tongyang Sun, \\ Quanquan Liu, Qing Shi, Jianjun Long, Yulong Wang, Zhengzhi Wu, Jian Qin, Weiguang Li, \\ Massimiliano Zecca, Atsuo Takanishi
}

\begin{abstract}
Nowadays, with the development of the proportion of the elderly population in the world, several problems caused by the population aging gradually into people's horizons. One of the biggest problems plagued the vast majority of the elderly is hemiplegia, which leads to the vigorous development of the physical therapists. However, these traditional methods of physical therapy mainly rely on the skill of the physical therapists. In order to make up the defects of traditional methods, many research groups have developed different kinds of robots for lower limb rehabilitation training but most of them can only realize passive training which cannot adopt rehabilitation training based on the patients' individual condition effectively and they do not have a rehabilitation evaluation system to assess the real time training condition of the hemiplegic patients effectively. In order to solve the problems above, this paper proposed a lower limb rehabilitation evaluation system which is based on the virtual reality technology. This system has an easy observation of the human-computer interaction interface and the doctor is able to adjust the rehabilitation training direct at different patients in different rehabilitation stage based on this lower limb rehabilitation evaluation system. Compared with current techniques, this novel lower limb rehabilitation evaluation system is expected to have significant impacts in medical rehabilitation robot field.
\end{abstract}

Shihui Shen, Chang Gao, Yong Zhao, Haojian Lu and Yajing Shen are affiliated with Department of Mechanical and Biomedical Engineering, City University of Hong Kong. (shihushen2@cityu.edu.hk)

Chunbao Wang works in the Shenzhen Second People's Hospital as a post doctor of Sun Yat-sen University. He is also a visiting researcher of Honkong City University and invited researcher of Waseda University. Address: Shenzhen Second People's Hospital, No. 3002, Sungang Xi Road, Shenzhen, 518037, P.R China. ( mrwang@fuji.waseda.jp)

Zhengzhi Wu is professor affiliated with Shenzhen Institute of Geriatrics and Shenzhen Second People's Hospital.

Atsuo Takanishi is with Mechanical Engineering Department, Waseda University, and is one of the core members of the Humanoid Robotics Institute (HRI), Waseda University (contact@takanishi.mech.waseda.ac.jp)

Massimiliano Zecca is the professor affiliated with Loughborough University(M.Zecca@lboro.ac.uk).

Jianjun Long, Yulong Wang are affiliated with Shenzhen Second People’s Hospital.

Quanquan Liu is affiliated with Shenzhen Institute of Geriatrics and the researcher of National University of Singapore

Qing Shi is affiliated with School of Mechatronical Engineering, Beijing Institute of Technology. He is also a visiting researcher of Takanishi Lab, Waseda University.

Jian Qin is a professor affiliated with the Fist Affiliated Hospital, Sun Yat-sen University.

Weiguang $\mathrm{Li}$ is a professor affiliated with South China University of Technology.

\section{INTRODUCTION}

Demographic ageing and many diseases caused by demographic ageing are key challenges facing societies during the twenty-first century [1-2]. As a result, age-related diseases have gradually become an emergency [3], of which hemiplegia is one of the biggest problems plagued the vast majority of the elderly [4]. Therefore, in order to make hemiplegia patients are able to recover gradually, it's important to arrange physical therapy and rehabilitation training for the patients [5].

Traditionally, hemiplegia patients may hire a physical therapist help then with rehabilitation training. As we know, this traditional method totally depend on the skill of the physical therapists [6-8]. Undoubtedly, a skilled physical therapist rehabilitation training can effectively help patients, shorten the recovery period, improve the efficiency of training and can make the hemiplegia patients recover as soon as possible. However, the level of the physical therapist is uneven, skilled therapist services are expensive and can only treat the patients one by one but the treatment of ordinary therapists cannot achieve the best effect or it may likely to delay recovery. Due to the limitation of the traditional rehabilitation training and with the rapid development of robot technology, lots of types of robots are developed by researchers to help patients with hemiplegia rehabilitation training [9-10].

Fraunhofer IPK automation robot research center from German developed an advanced mechanized gait trainer, which can simulate ankle walking trajectory based on able-bodied person [11]. The rehabilitation robot Kine Assist developed by University of Chicago can help patients control the barycenter during the walking and can also help patients with stand up training and squat training [12]. Researchers in Japan developed a rehabilitation robot called Gait Master, which composed of the rotary with three degrees of freedom. This robot can provide training under different road conditions for a patient [13]. Walking rehabilitation robot Lokomat developed by HOCOMA Company in Swiss can help the patients complete the reciprocating gait training in the sagittal plane [14]. Mechanical Engineering College and Medical College in University of Delaware jointly developed a lower limb exoskeleton gait orthoses with gravity balance system [15]. What's more, other institutions around the world also have developed many types of medical rehabilitation robot with various functions [16-20].

However, most robot systems mentioned above are lack of active rehabilitation training and they do not have a 
rehabilitation evaluation system to assess the real time training condition of the hemiplegic patients effectively. In order to solve the problems above, this paper proposed a lower limb rehabilitation evaluation system which is based on the virtual reality technology. This system has an easy observation of the human-computer interaction interface and the doctor is able to adjust the rehabilitation training direct at different patients in different rehabilitation stage based on this lower limb rehabilitation evaluation system. The rest of this paper is organized as follows. Section II gives an introduction of rehabilitation evaluation system setup which is composed of the LP-RESEARCH Motion Sensor (LPMS) and the human-computer interaction interface based on virtual reality technology. Section III addresses the principle of the motion tracking system which is composed of iterative skeletal model and the software structure. Section IV shows the experimental results. Finally, Section V offers conclusions to this paper and discusses the potential applications and long-term impacts of this rehabilitation evaluation system.

\section{ReHABILITATION EVALUATION System SETUP}

This rehabilitation evaluation system mainly has two parts, the one is motion tracking part which mainly consists of several sensors, and the other is human-computer interaction interface which is based on Unity Engine. The following of this section will mainly introduce these two parts.

\section{A. LP-RESEARCH Motion Sensor (LPMS)}

In order to clearly express the motion and the angle of each joint of the hemiplegic patients' lower limbs during rehabilitation training. The LP-RESEARCH Motion Sensor (LPMS) is adopted as the motion sensor. This type of sensor is a miniature, multi-purpose inertial measurement unit which can measure orientation in 360 degrees about all three global axes. We choose LPMS-B as our sensor and the main specifications of this sensor are shown in TABLE I.

TABLE I

\begin{tabular}{|l|l|}
\hline Unit type & LPMS-B \\
\hline Interface type & Bluetooth 2 \\
\hline Maximum baud rate & 9216000 Baud \\
\hline Communication protocol & LPBUS \\
\hline Size & $45^{*} 37 * 20 \mathrm{~mm}$ \\
\hline Weight & $34 \mathrm{~g}$ \\
\hline Bluetooth & $2.1+\mathrm{EDR} 2.412-2.484 \mathrm{GHz}$ \\
\hline Communication distance & $<18 \mathrm{~m}$ \\
\hline Maximum data transmission rate & $133 \mathrm{~Hz}$ \\
\hline Latency & $15 \mathrm{~ms}$ \\
\hline Power consumption & $290 \mathrm{~mW} @ 3.3 \mathrm{~V}$ \\
\hline Power supply & Lithium battery $>10 \mathrm{~h}(3.7 \mathrm{~V}$ \\
@800mAh)
\end{tabular}

The measurements of this sensor can be taken digitally and transmitted to a data analysis system in the form of orientation quaternion or Euler angles. This sensor calculates the orientation difference between a fixed sensor coordinate system and a global reference coordinate system. The local and the global reference coordinate systems used are defined as right handed Cartesian coordinate system with: $\mathrm{X}$ positive when pointing to the magnetic west, $\mathrm{Y}$ positive when pointing to the magnetic south and $\mathrm{Z}$ positive when pointing up (gravity points vertically down with-1g).

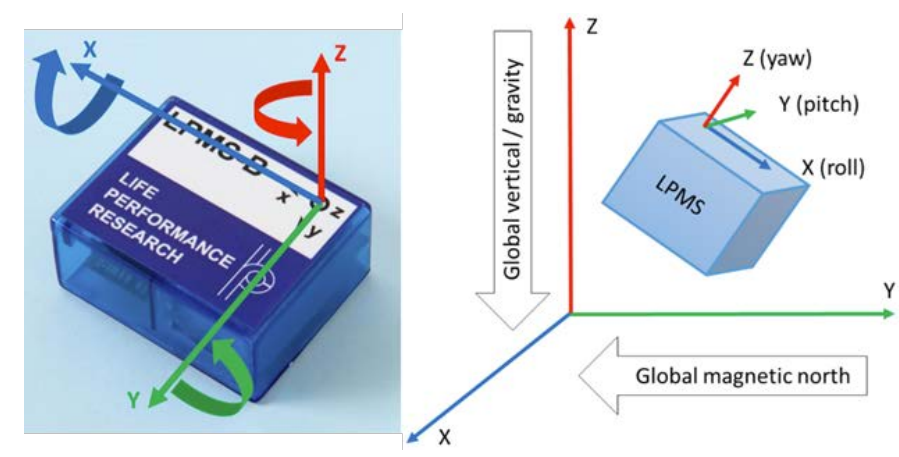

Fig.1 The orientation and relationship of local sensor and earth global coordinate system

As shown in Fig.1, the orientation and relationship of local sensor and earth global coordinate system can be observed by researchers. What's more, the 3D orientation output is defined as the orientation between the body-fixed coordinate system and the global coordinate system, using the global coordinate system as reference.

\section{B. Human-computer Interaction Interface}

In this paper, Unity Engine is used to construct the virtual reality. Unity is a cross-platform game engine developed by Unity Technologies and used to develop video games for PC, consoles, mobile devices and websites.

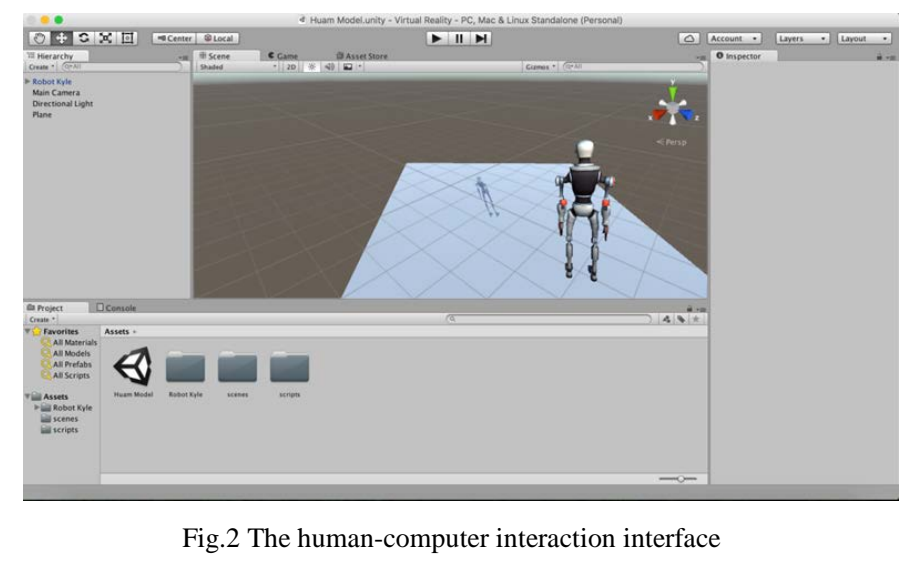

As shown in Fig.2, a human-computer interaction interface is designed based on virtual reality technology. The main purpose of building this platform is realizing real-time display of the motion of hemiplegic patients so that the doctor can observe the condition of the patients during the rehabilitation training. If something wrong happen during the patients' rehabilitation training, the doctor can find the problem in a timely manner and make corresponding adjustment.

\section{Principle of The Motion Tracking System}

After the rehabilitation evaluation system setup, we need to address the principle of the motion tracking system which is composed of iterative skeletal model and the software 
structure. The iterative skeletal model is proposed to transfer the sensor data into the motion of hemiplegia patients and the software structure is proposed to connect the motion of hemiplegia patients with the model in the human-computer interaction interface based on virtual reality technology.

\section{A. Iterative Skeletal Model}

In this paper, we intend to figure out the orientation of human body, such as how shank rotated when human is walking. If the orientation of whole human body can be sensed, it is desired to develop a system to rebuild the human motion model in virtual reality environment where the virtual model can tracking the motion of human in real-time. Furthermore, the orientation data is recorded as the real-time processing of virtual model so that an evaluation system should be developed based on the data of orientation. In spite of how the evaluation system working, the method of retrieving orientation information of human body should be researched at first.

Based on the wireless bluetooth characteristics, it is convenient to retrieve the quaternion data through bluetooth connection with host machine, in this case, which is PC. The quaternion, which is elaborated in previous chapter, indicates that it is possible to recognize how the sensor titled. Assuming we have a rigid body, now attaching wireless bluetooth sensor with its $\mathrm{Y}$ axis aligning the direction of this rigid body. It is obvious that wherever $\mathrm{Y}$ axis points is the rigid body points. This is the intuitive idea of bone orientation sensing method

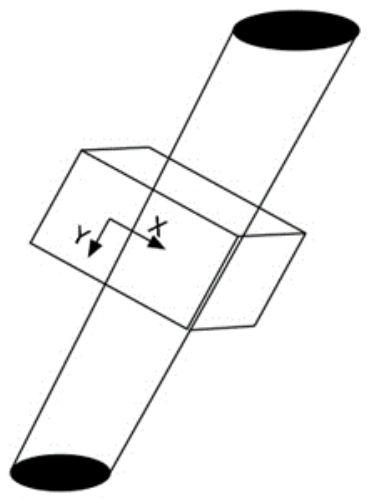

Fig. 3 The model of a rigid body with sensor fixed aside

In order to further develop the sensing method to adapt the situation of human body area, a set of sensor placement is established. As shown in Fig.3, Y axis aligns the direction of limb and $\mathrm{X}$ axis is in horizontal direction so that $\mathrm{Z}$ axis against towards human horizontally. However, the sensor output is the rotation form global coordinate to local coordinate, the initial human limb's posture does not necessarily align with the global coordinate. As the result of above issue, the output of sensor should not be used directly for representing limb orientation. Instead, it is proper to use the rotation changes from initial output and the output at Time t. In other words, if we preset the attention posture as the initial posture, the sensor output of rotation after that initial posture is the rotation information that drive the model.
Now we try to use sensor to rotate a rigid body, assuming the rigid body as a vector, which is represented in terms of quaternion with real part equals to zero.

$$
v_{0}=0+u
$$

To rotate the vector using the quaternion $\mathrm{q}$, which is the rotation between attention posture and posture at Time t, as shown in eq.2.

$$
v_{1}=q * v_{0} * q
$$

When the sensor is setup, the output of sensor is the rotation from global coordinate to its initial posture. However, when the sensor is placed on human body with attention posture, an attention quaternion should be record, which is $\mathrm{q}_{0}$. Corresponding, $\mathrm{q}_{1}$ is another quaternion that represent the rigid body rotation based on the data retrieved from sensor at Time t. Noted that q should be the rotation between attention posture and Time t posture, as shown in eq.3 and eq.4.

$$
\begin{aligned}
& q_{2}=q * q_{1} \\
& q=q_{2} * q_{0}{ }^{-1}
\end{aligned}
$$

The scenario is that the attention posture is preset and to drive the model in virtual reality environment, human body is necessary to start moving form attention posture, which implies that the more the real world human attention posture is close to virtual attention posture, the more precision will be achieved.

As mentioned above, it is feasible to sense the orientation of a single bone in term of sensor property placed on human body. Beside for single one bone, it is intuitively consider using a set of sensors to setup a system to capture whole body motions. According Havanan's model, as shown in Fig. 4, the human body was represented as stick figure, a human virtual rendering standards model articulated structure using segments and joints. The body segments could be approximated as consecutive lines.

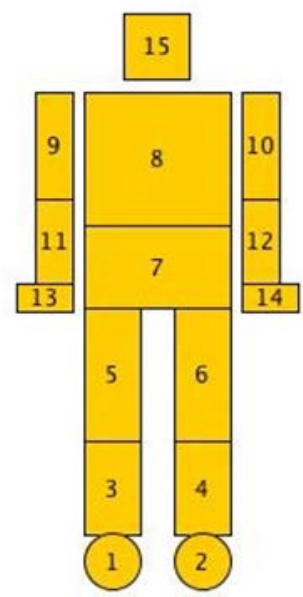

Fig.4 Havanan's model of a human body

Now based on one single bone's orientation, applying iterative model to the base bone. By iterative, assuming one bone has base point and tip point, means that for a bone its base point is determined by its former bone's tip point. From the Base to the tip of one single bone and then extend to another bone's base point. 

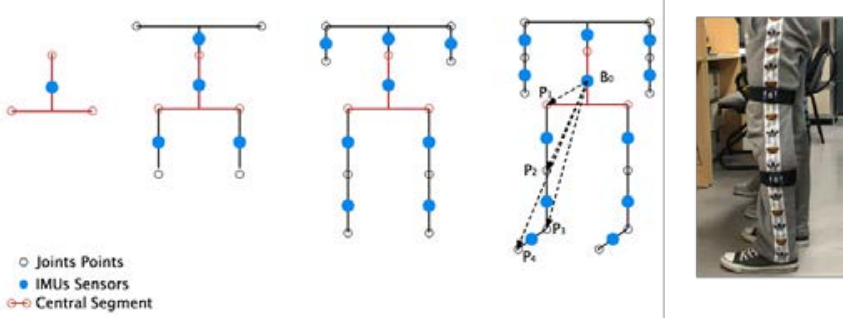

Fig.5 Simplified skeletal model with sensors

As shown in Fig.5, human body is model as 12 segments. Each segment is can be model as rigid body. These rigid body were connected form central segment to tip segment. So that the calculation starts from central base, each bone is model as a vector, which is rotated by the quaternion sensed by the sensor attached on corresponding bone.

$$
\begin{gathered}
q_{n t}=q_{n} q_{\text {int }} \\
q_{n t} q_{\text {int }}{ }^{-1}=q_{n} \\
\overrightarrow{B_{0} P_{1}}=q_{0} \overrightarrow{B_{0} P_{1 i n t}} q_{0}{ }^{-1} \\
\overrightarrow{B_{0} P_{2}}=\overrightarrow{B_{0} P_{1}}+q_{1} \overrightarrow{P_{1} P_{2 i n t}} q_{1}^{-1} \\
\overrightarrow{B_{0} P_{3}}=\overrightarrow{B_{0} P_{2}}+q_{2} \overrightarrow{P_{2} P_{3 i n t}} q_{2}^{-1} \\
\overrightarrow{B_{0} P_{4}}=\overrightarrow{B_{0} P_{3}}+q_{3} \overrightarrow{P_{3} P_{4 i n t}} q_{3}^{-1}
\end{gathered}
$$

Given that all the bones are connected, the position of the tip of one bone will be the base position of the next bone. Furthermore, based the eq.5 - eq.10, if given the position of central based point $\mathrm{B}_{0}$, the position of each bone is calculated. Using this we can sequentially calculate the positions of the bones from one to the next like a chain, updating all of their positions appropriately.

In case of measuring the joint angle, the model is needed to be more specific in terms of placement. Generally speaking, to rotate the bone from attention posture, there are two prerequisites, which are attention posture and Time $\mathrm{t}$ quaternion, as shown in eq.4.

Note that $\mathrm{q}$ is the rotation from one posture to another. Considering to replace $q 1$ with the quaternion of one bone and q2 with another bone which directly connects to former bone. It turns out that the $q$ becomes now the posture form one bone to another.

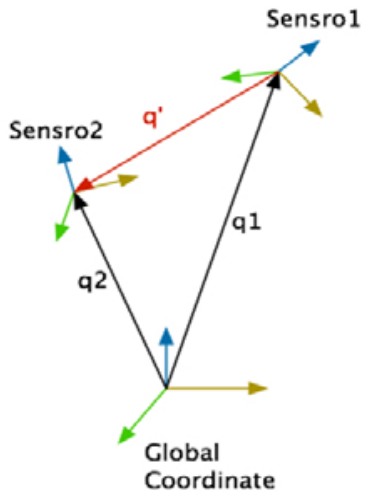

Fig.6 The relationship between two sensors
As shown in Fig. 6, it is obvious that the $q$ from this scenario is the rotation form one sensor to another, which implies that it is not necessary to present the rotation angle on bones, as long as the placement of sensor are proper. By proper, in the situation of attention posture, two sensors are necessary to placed uniformly so that the rotation of joint is captured by the difference output of two sensors.

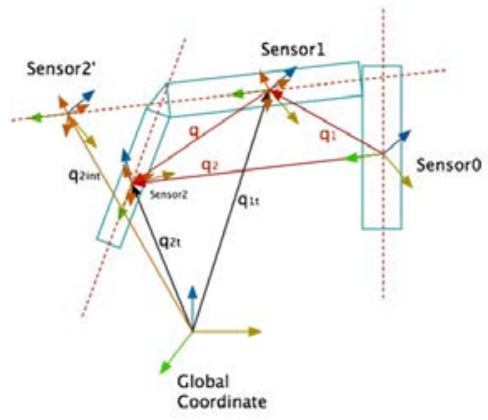

Fig.7 Three segments model

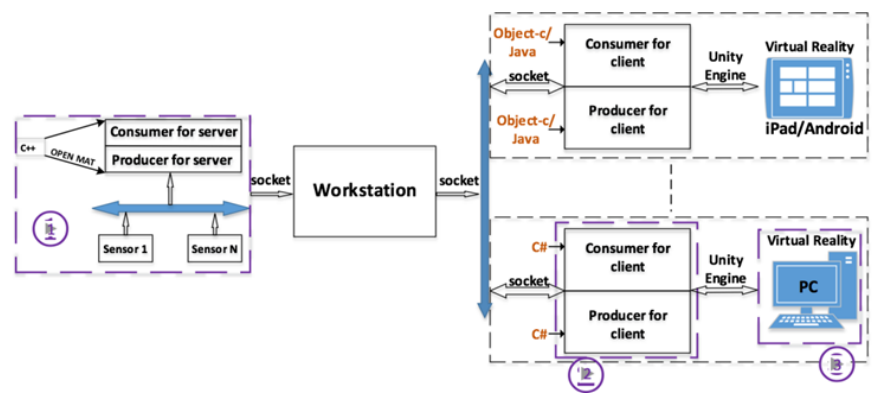

Fig.8 Software Structure

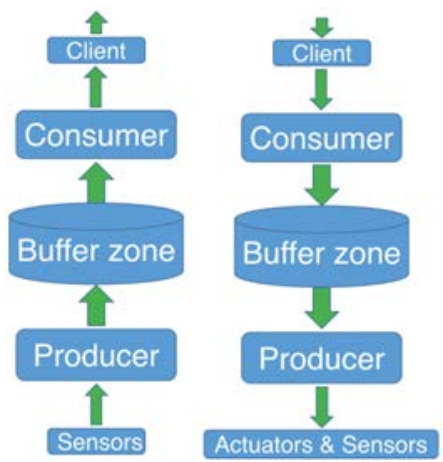

Fig.9 The framework of Producer-Consumer mode

The measurement model is then further extend to three segments. Based the model of two segments, in three situation, it is intuitively to know that calculate the $\mathrm{q}$ of each sensor respectively and then calculate the difference in between.

\section{B. Software Structure}

As shown in Fig.8, the software structure is mainly composed of two parts, the first one is server collecting data from sensor which is developed by $\mathrm{C}++$ and the second is client which is drive the model moving according to human's motions. The developing software is Visual Studio 2015 and Unity3D, respectively.

In order to affirm the stability of transmitting data, we use the producer-consumer mode to transmit data, as shown in 
Fig.9. This mode has several advantages, it can confirm data stabilization, has multiple threads so that the speed which producer makes data does not depend on the speed which consumer use data and vice versa and can be decoupling so that when changing the code of producer, we do not have to change the code of consumer and vice versa.

\section{EXPERIMENTAL RESULTS}

In order to examine the performance of the lower limb rehabilitation evaluation system, experiments have been conducted through rehabilitation training shown in Fig.10.

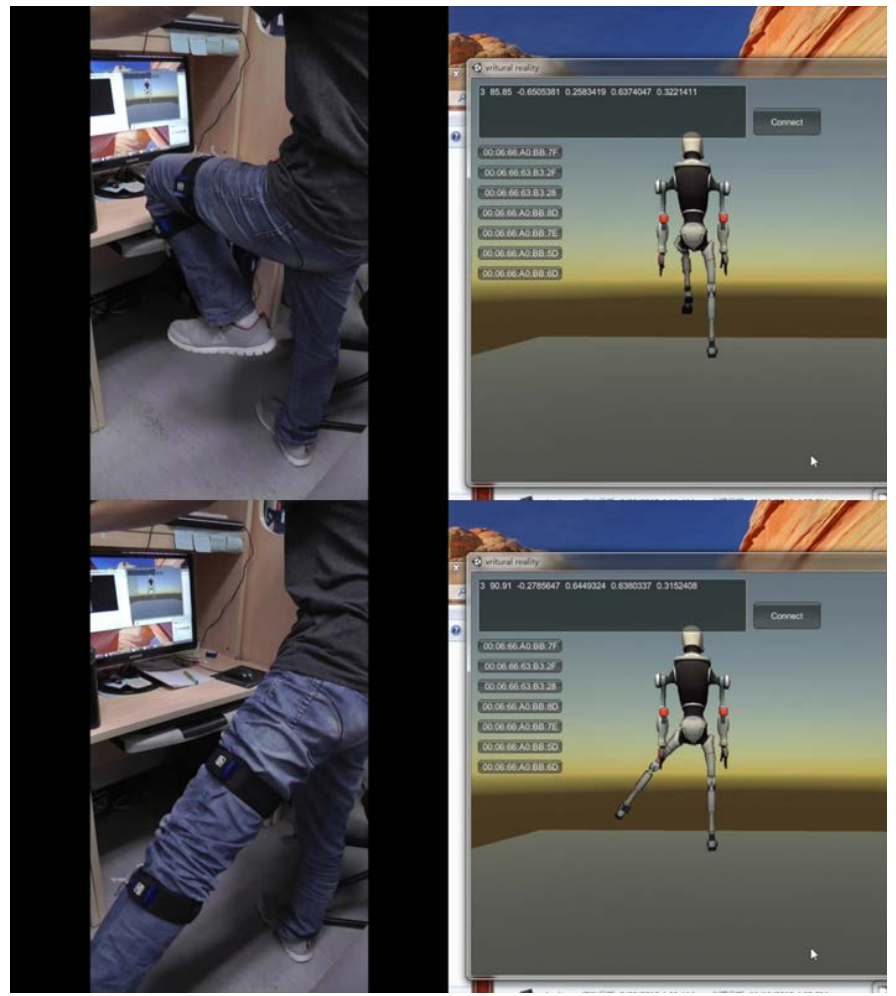

Fig.10 The experimental system

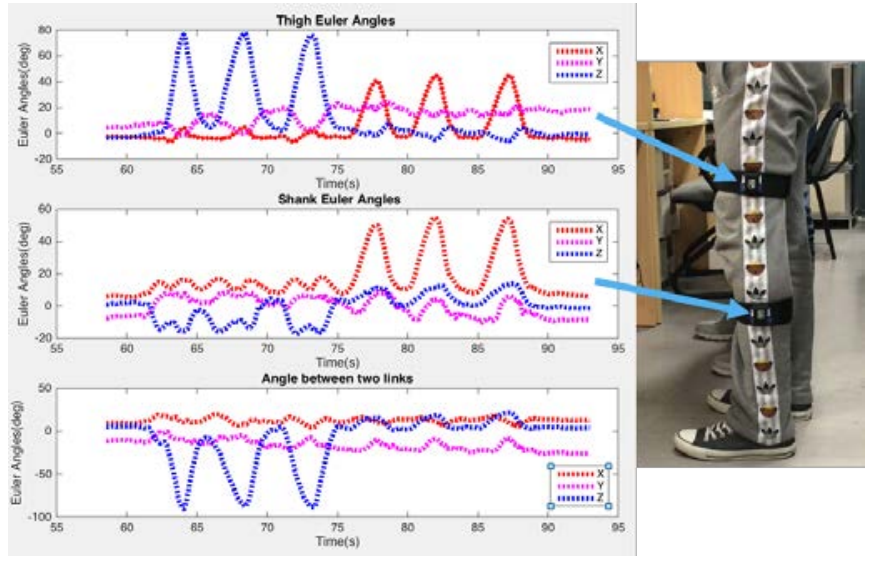

Fig.11 Experimental results

Given the human iterative model elaborated in previous section, we conduct two experiments, which one is for three sensors rotation simulation and another is for three sensors rotation on human body. The principle within the simulation experiment is that under uniform installation, the sensor will strictly rotated around a single axis so that the output can be perceived as simple pattern.

Now in this experiment, sensors are attached on human body, as shown in Fig.11. As long as sensors are all installed uniformly the output should be very similar to the previous simulation experiment, which indicates the angle of sensors. However on human installed experiment, because of the installment errors, which is caused by muscle elasticity, the initial output of the sensor are non-zero so that it will slightly influence the output of other axis when human is working. The first figure indicates that the sensor at first rotates around $\mathrm{Z}$ axis and then rotates around $X$ axis, meanwhile the oscillating of other axis can be perceived when a single axis is rotating. The same as second figure. However, when lift leg in front the shank indeed will not rotate as dramatically as thigh, which can be perceived in second figure. But when lift leg on side direction with leg keeping stretched, the $\mathrm{X}$ axis rotation can be record simultaneously. In third figure, the fusion of previous two sensors is achieved and the significant rotation of $\mathrm{Z}$ axis between Thigh and Shank sensors is perceived.

Based on all the experimental results mentioned above, it seems that this novel lower limb rehabilitation evaluation system is practical and reliable. It meets the demands of an easy observation of the human-computer interaction interface and the doctor is able to adjust the rehabilitation training direct at different patients in different rehabilitation stage based on this system.

\section{CONCLUSION}

Due to current medical rehabilitation robot systems lack of rehabilitation evaluation system to assess the real time training condition of the hemiplegic patients effectively, this paper proposed a novel lower limb rehabilitation evaluation system which is based on the virtual reality technology. This system has an easy observation of the human-computer interaction interface and the doctor is able to adjust the rehabilitation training direct at different patients in different rehabilitation stage based on this lower limb rehabilitation evaluation system. To validate the proposed system, we have done the experiment via rehabilitation training on human body. Through the experiments, this rehabilitation evaluation system can complete the task successfully, so it is practical and reliable.

In our next work, we will finish the whole human body iterative skeletal modeling and whole human body model virtualization. This research is expected to have significant impacts in medical rehabilitation robot field.

\section{ACKNOWLEDGMENT}

*Resrach supported by ITC Platform Research Projects 9440144, Shenzhen-Honkong Joint Research Project SGLH20150211093607169, and China Post-doctor Research Foundation 2015M580759.

\section{REFERENCES}

[1] Reher D S. Baby booms, busts, and population ageing in the developed world[J] Population studies, 2015, 69(sup1): S57-S68. 
[2] Bonney A, Phillipson L, Jones S C, et al. The brave new world of older patients: preparing general practice training for an ageing population[J]. Primary health care research \& development, 2015: 1-11.

[3] Dong L, Xiao R, Cai C, et al. Diet, lifestyle and cognitive function in old Chinese adults[J]. Archives of Gerontology and Geriatrics, 2016, 63: 36-42.

[4] Giaquinto S, Majolo I, Palma E, et al. Very old people can have favorable outcome after hip fracture: 58 patients referred to rehabilitation[J]. Archives of gerontology and geriatrics, 2000, 31(1): 13-18.

[5] Jishu Rong. Practical hemiplegia rehabilitation technical illustration. People’s Medical Publishing Housh, 2015.

[6] Lu Z, Wang C, Duan L, et al. Development of a novel ankle rehabilitation robot with three freedoms for ankle rehabilitation training[C]//Cyber Technology in Automation, Control, and Intelligent Systems (CYBER), 2015 IEEE International Conference on. IEEE, 2015: 2091-2096.

[7] Wang C, Wang L, Qin J, et al. Devel.

[8] opment of an ankle rehabilitation robot for ankle training[C]//Information and Automation, 2015 IEEE International Conference on. IEEE, 2015: 94-99.

[9] Sun T, Lu Z, Wang C, et al. Mechanism design and control strategies of an ankle robot for rehabilitation training[C]//Robotics and Biomimetics, 2015 IEEE International Conference on. IEEE, 2015: 132-137.

[10] Wang C, Lu Z, Wang Y, et al. Development of a rehabilitation robot for hand and wrist rehabilitation training[C]//Information and Automation, 2015 IEEE International Conference on. IEEE, 2015: 106-111.

[11] $\mathrm{Lu} \mathrm{Z,} \mathrm{Li} \mathrm{W,} \mathrm{Li} \mathrm{M,} \mathrm{et} \mathrm{al.} \mathrm{Development} \mathrm{of} \mathrm{a} \mathrm{three} \mathrm{freedoms} \mathrm{ankle}$ rehabilitation robot for ankle training[C]//TENCON 2015-2015 IEEE Region 10 Conference. IEEE, 2015: 1-5.

[12] Hesse S, Uhlenbrock D. A mechanized gait trainer for restoration of gait[J]. Journal of rehabilitation research and development, 2000, 37(6): 701-708.

[13] Burgess J K, Weibel G, Brown D. Increases in overground gait speed with body weight support in people post-stroke[C]//Rehabilitation Robotics, 2009. ICORR 2009. IEEE International Conference on. IEEE, 2009: 401-406.

[14] Furusho J. Mechatronics system using ER fluids[J]. Journal of Japan Hydraulics and Pneumatic Society, 2001, 32(6): 390-395.

[15] Riener R, Lünenburger L, Colombo G. Human-centered robotics applied to gait training and assessment[J]. Journal of rehabilitation research and development, 2006, 43(5): 679.

[16] Verrelst B, Van Ham R, Vanderborght B, et al. The pneumatic biped "Lucy" actuated with pleated pneumatic artificial muscles[J]. Autonomous Robots, 2005, 18(2): 201-213.

[17] Girone M, Burdea G, Bouzit M, et al. A Stewart platform-based system for ankle telerehabilitation[J]. Autonomous robots, 2001, 10(2): 203-212.

[18] Saglia J, Tsagarakis N G, Dai J S, et al. A high performance 2-dof over-actuated parallel mechanism for ankle rehabilitation[C]//Robotics and Automation, 2009. ICRA'09. IEEE International Conference on. IEEE, 2009: 2180-2186.

[19] Yoon J, Ryu J. A novel reconfigurable ankle/foot rehabilitation robot[C]//Robotics and Automation, 2005. ICRA 2005. Proceedings of the 2005 IEEE International Conference on. IEEE, 2005: 2290-2295.

[20] Saglia J A, Tsagarakis N G, Dai J S, et al. A high performance redundantly actuated parallel mechanism for ankle rehabilitation[J]. The International Journal of Robotics Research, 2009.

[21] Gosselin C M, Lavoie E. On the kinematic design of spherical three-degree-of-freedom parallel manipulators[J]. The International Journal of Robotics Research, 1993, 12(4): 394-402. 